\title{
Clinical significance of mucinous component in colorectal adenocarcinoma: a propensity score-matched study
}

\author{
Chuanwang Yan ${ }^{1 \dagger}$, Hui Yang ${ }^{2 \dagger}$, Lili Chen ${ }^{3 \dagger}$, Ran Liu ${ }^{2}$, Wei Shang ${ }^{2}$, Wenguang Yuan ${ }^{2}$, Fei Yang ${ }^{3 *}$, Qing Sun ${ }^{4^{*}}$ and \\ Lijian $\mathrm{Xia}^{3^{*}}$
}

\begin{abstract}
Background: This study aims to investigate the clinical significance and prognostic value of mucinous component $(\mathrm{MC})$ in colorectal adenocarcinoma (AC).

Methods: Patients with colorectal AC and AC with MC (ACMC) (1-100\%) underwent surgical resection between January 2007 and February 2018 were retrospectively reviewed. Propensity score matching (PSM) was performed according to a 1:1 ratio. Receiver-operating characteristic (ROC) curve was used to identify the optimal cut-off value of $\mathrm{MC}$ ratio for prognostic prediction. The clinicopathological features and 3-year overall survival (OS) of AC patients, mucinous adenocarcinoma (MAC) (MC > 50\%) patients, and ACMC (1-50\%) patients were compared before and after matching. Multivariable analysis was used for analyzing independent risk factors related to prognosis.

Results: A total of 532 patients were enrolled in this study. Patients with AC, MAC, and ACMC (1-50\%) exhibited different clinicopathological features. However, their 3 -year OS rates were similar $(82.00 \%$ vs. $74.11 \%$ vs. $81.48 \%, P=0.38)$. After matching, ROC curve determined $70 \%$ as the optimal cut-off value. And patients with ACMC $>70 \%$ had a much poorer 3-year OS compared with ACMC (1-70\%) patients and AC patients (47.37\% vs. 86.15\% vs. $79.76 \%, P<0.001)$. In addition, $A C M C>70 \%$ was revealed as a risk factor for poor survival in univariate analysis $(\mathrm{HR}=1.643,95 \% \mathrm{Cl}=1.025-$ $2.635, P=0.039)$, though not an independent risk factor in multivariable analysis $(\mathrm{HR}=1.550,95 \% \mathrm{Cl}=0.958-2.507$, $P=0.074)$.
\end{abstract}

Conclusions: $M A C$ is usually diagnosed at an advanced stage. MAC has a similar survival with AC and ACMC (1-50\%) patients before and after matching. Patients with ACMC > 70\% exhibited a much poorer OS, and should be given more clinical attention.

Keywords: Colorectal cancer, Adenocarcinoma, Mucinous component, Survival prognosis

*Correspondence: yangf-2008@163.com; qingsw99@sdhospital.com.cn; xiaalbert2758@163.com

${ }^{\dagger}$ Yan Chuanwang, Yang Hui and Chen Lili contributed equally to this work. ${ }^{3}$ Department of Pathology, Jinan Central Hospital Affiliated to Shandong First Medical University, Jinan 250000, Shandong, China

${ }^{4}$ Department of Pathology, The First Affiliated Hospital of Shandong First Medical University \& Shandong Provincial Qianfoshan Hospital, Shandong Medicine and Health Key Laboratory of Clinical Pathology, Shandong Lung Cancer Institute, Shandong Institute of Nephrology, Jinan, China Full list of author information is available at the end of the article

\section{Introduction}

Colorectal cancer (CRC) ranks the world's fourth most deadly cancer with almost 900,000 deaths annually [1]. CRC has several histological types, and mucinous adenocarcinoma (MAC) comprises about $1.6-25.4 \%$ of all CRC cases [2]. According to the World Health Organization (WHO) criteria, MAC is defined as "> $50 \%$ of the lesion is composed of pools of extracellular mucin that contain malignant epithelium" [3]. However, $50 \%$ is more a cutoff 
Table 1 Clinicopathological parameters for patients before matching

\begin{tabular}{|c|c|c|c|c|}
\hline Clinicopathological parameters & Adenocarcinoma & $\begin{array}{l}\text { Adenocarcinoma with mucinous } \\
\text { component }(1-50 \%)\end{array}$ & $\begin{array}{l}\text { Mucinous } \\
\text { adenocarcinoma (>50\%) }\end{array}$ & $P$ \\
\hline Gender & & & & 0.501 \\
\hline Female & 174 & 25 & 18 & \\
\hline Male & 265 & 29 & 21 & \\
\hline Age & & & & 0.150 \\
\hline$<60$ years & 13 & 12 & 16 & \\
\hline$\geq 60$ years & 306 & 42 & 23 & \\
\hline Smoking & & & & 0.238 \\
\hline No & 328 & 44 & 33 & \\
\hline Yes & 111 & 10 & 6 & \\
\hline Alcoholism & & & & 0.215 \\
\hline No & 332 & 46 & 32 & \\
\hline Yes & 107 & 8 & 7 & \\
\hline ASA class & & & & 0.162 \\
\hline$\|$ & 354 & 38 & 29 & \\
\hline III & 85 & 16 & 10 & \\
\hline Hypertension & & & & 0.952 \\
\hline No & 311 & 39 & 27 & \\
\hline Yes & 128 & 15 & 12 & \\
\hline Diabetes mellitus & & & & 0.691 \\
\hline No & 381 & 46 & 32 & \\
\hline Yes & 58 & 8 & 7 & \\
\hline CAD & & & & 0.386 \\
\hline No & 398 & 46 & 34 & \\
\hline Yes & 41 & 8 & 5 & \\
\hline Hepatitis & & & & 0.821 \\
\hline No & 433 & 53 & 38 & \\
\hline Yes & 6 & 1 & 1 & \\
\hline CEA & & & & 0.114 \\
\hline$<5 \mathrm{ng} / \mathrm{ml}$ & 302 & 30 & 24 & \\
\hline$\geq 5 \mathrm{ng} / \mathrm{ml}$ & 137 & 24 & 15 & \\
\hline CA19-9 & & & & 0.006 \\
\hline$<37 \mathrm{U} / \mathrm{ml}$ & 393 & 42 & 30 & \\
\hline$\geq 37 \mathrm{U} / \mathrm{ml}$ & 46 & 12 & 9 & \\
\hline Albumin & & & & 0.006 \\
\hline$<40 \mathrm{~g} / \mathrm{dl}$ & 155 & 30 & 19 & \\
\hline$\geq 40 \mathrm{~g} / \mathrm{dl}$ & 284 & 24 & 20 & \\
\hline $\mathrm{HGB}$ & & & & 0.007 \\
\hline$<110 \mathrm{~g} / \mathrm{L}$ & 90 & 18 & 15 & \\
\hline$\geq 110 \mathrm{~g} / \mathrm{L}$ & 349 & 36 & 24 & \\
\hline Occult blood & & & & 0.011 \\
\hline No & 145 & 27 & 19 & \\
\hline Yes & 294 & 27 & 20 & \\
\hline Operation time & & & & 0.738 \\
\hline$<3 h$ & 197 & 25 & 20 & \\
\hline$\geq 3 h$ & 242 & 29 & 19 & \\
\hline Perioperative blood transfusion & & & & 0.851 \\
\hline No & 333 & 41 & 28 & \\
\hline Yes & 106 & 13 & 11 & \\
\hline Tumor location & & & & $<0.001$ \\
\hline
\end{tabular}


Table 1 (continued)

\begin{tabular}{|c|c|c|c|c|}
\hline Clinicopathological parameters & Adenocarcinoma & $\begin{array}{l}\text { Adenocarcinoma with mucinous } \\
\text { component }(1-50 \%)\end{array}$ & $\begin{array}{l}\text { Mucinous } \\
\text { adenocarcinoma (>50\%) }\end{array}$ & $P$ \\
\hline Right-sided & 43 & 16 & 13 & \\
\hline Left-sided & 396 & 38 & 26 & \\
\hline Defunctioning stoma & & & & 0.705 \\
\hline No & 434 & 53 & 39 & \\
\hline Yes & 5 & 1 & 0 & \\
\hline Postoperative complication & & & & 0.635 \\
\hline Absent & 374 & 46 & 31 & \\
\hline Present & 65 & 8 & 8 & \\
\hline Differentiation & & & & $<0.001$ \\
\hline Well/Moderate & 368 & 37 & 18 & \\
\hline Poor & 71 & 17 & 21 & \\
\hline Signet-ring cell component & & & & 0.341 \\
\hline Absent & 436 & 54 & 38 & \\
\hline Present & 3 & 0 & 1 & \\
\hline $\mathrm{PNI}$ & & & & 0.735 \\
\hline Yes & 24 & 3 & 1 & \\
\hline No & 415 & 51 & 38 & \\
\hline LVI & & & & 0.551 \\
\hline Yes & 54 & 8 & 7 & \\
\hline No & 385 & 46 & 32 & \\
\hline T stage & & & & $<0.001$ \\
\hline $1 / 2$ & 138 & 7 & 3 & \\
\hline $3 / 4$ & 301 & 47 & 36 & \\
\hline N stage & & & & 0.240 \\
\hline 0 & 224 & 30 & 15 & \\
\hline $1 / 2$ & 215 & 24 & 24 & \\
\hline TNM stage & & & & 0.240 \\
\hline$|/| \mid$ & 224 & 30 & 15 & \\
\hline III & 215 & 24 & 24 & \\
\hline
\end{tabular}

ASA American Society of Anesthesiologists, CAD coronary artery disease, CEA carcinoembryonic antigen, CA19-9 carbohydrate antigen 19-9, HGB hemoglobin, TNM tumor-lymph node-metastasis, LVI lymphovascular invasion, $P N I$ perineural invasion

value defining MAC pathologically than a clinical index indicating clinical significance and prognosis. An optimal cut-off value of mucinous proportion better defining its clinical significance is needed.

Previous studies have discovered that MAC was associated with young age, advanced tumor stage, accumulation in female patients, and distinct molecular patterns, such as microsatellite instability and activating mutations of the BRAF gene $[2,4,5]$. When analyzing clinical outcomes, the clinicopathological differences between MAC and $\mathrm{AC}$ are potential confounding factors. At present, findings regarding the progressive behavior and survival remain controversial in MAC [6]. Due to the lack of substantiated data, MAC specialized treatment strategy remains unclear and patients with MAC are usually treated along the lines of recommendations for adenocarcinoma (AC) of the CRC [7]. Thus, more solid evidence is needed to evaluate the significance of mucinous component $(\mathrm{MC})$ in $\mathrm{AC}$.

The present study aims to further evaluate the clinical significance and prognostic value of MC in AC. Slides of $\mathrm{AC}$ with $\mathrm{MC}$ (ACMC) (1-100\%) were reviewed, and proportion of $\mathrm{MC}$ in $\mathrm{AC}$ was re-evaluated. Propensity-score matched (PSM) analysis was conducted to minimize bias. The optimal cut-off value of the MC proportion for prognostic prediction was analyzed. The clinicopathological features and survival of enrolled cases were also depicted before and after matching. Meanwhile, the potential risk factors for poor survival were identified.

\section{Materials and methods}

Study population

Records of CRC patients underwent surgical resection from January 2007 to February 2018 at the First Affiliated 
Hospital of Shandong First Medical University \& Shandong Provincial Qianfoshan Hospital were reviewed. Final diagnosis was confirmed by pathology. Patients with a history of cancer, two or more cancers, synchronous distant metastasis, local excision, palliative surgery, and no complete clinicopathological or follow-up data were excluded. We collected the following data of each patient from clinical records: gender, age, history of smoking and alcoholism, the American Society of Anesthesiologists (ASA) class, comorbidities (hypertension, diabetes mellitus, coronary artery disease (CAD), and hepatitis), preoperative carcinoembryonic antigen (CEA), carbohydrate antigen 19-9 (CA19-9), albumin, and hemoglobin (HGB) levels, occult blood status, operative factors (operation time, perioperative blood transfusion, defunctioning stoma, and postoperative complications), and tumor factors (tumor location, differentiation, signet-ring cell component, perineural invasion (PNI), lymphovascular invasion (LVI), T stage, $\mathrm{N}$ stage, $\mathrm{M}$ stage and TNM stage). Written informed consent was signed by each patient. This study was approved by the First Affiliated Hospital of Shandong First Medical University \& Shandong Provincial Qianfoshan Hospital Institutional Review Board.

\section{Follow-up method}

Patients were followed up postoperatively every 6 months for 2 years, and then annually for 3-5 years at outpatient clinic. Physical examination, serum tumor markers, including CEA, and abdominal/chest/pelvic imaging using a CT scan were used for surveillance. Colonoscopy was performed at the 1st and 2nd year after surgery. Overall survival (OS) was defined as the period from the surgery to death from any cause.

\section{Pathological evaluation}

For each case, the number of paraffin block for pathological evaluation was determined based on the tumor size (1 block per $\mathrm{cm}$ ). Tumor sections from paraffin blocks were stained with hematoxylin-eosin. The ratio of MC area was separately evaluated by two pathologists, and the mean value was adopted. If the difference in estimated values was $10 \%$ or greater, the two pathologists reassessed the specimens to determine the consensus. Finally, tumors, with $\mathrm{MC}$ proportion ranging from 1 to $100 \%$, were classified into 10 groups evenly with $10 \%$ ingredient per group. Classical gland-forming adenocarcinomas with variable size and configuration of the glandular structures were classified as AC. ACMC was defined as tumors with $1-100 \%$ of the lesion being composed of mucin, typically characterized by pools of extracellular mucin that contain malignant epithelium as acinar structures, strips of cells, or single cells. And those with more than 50\% mucin in tumor were labelled as MAC. Signet ring cell component was defined as $\mathrm{AC}$ with signet ring
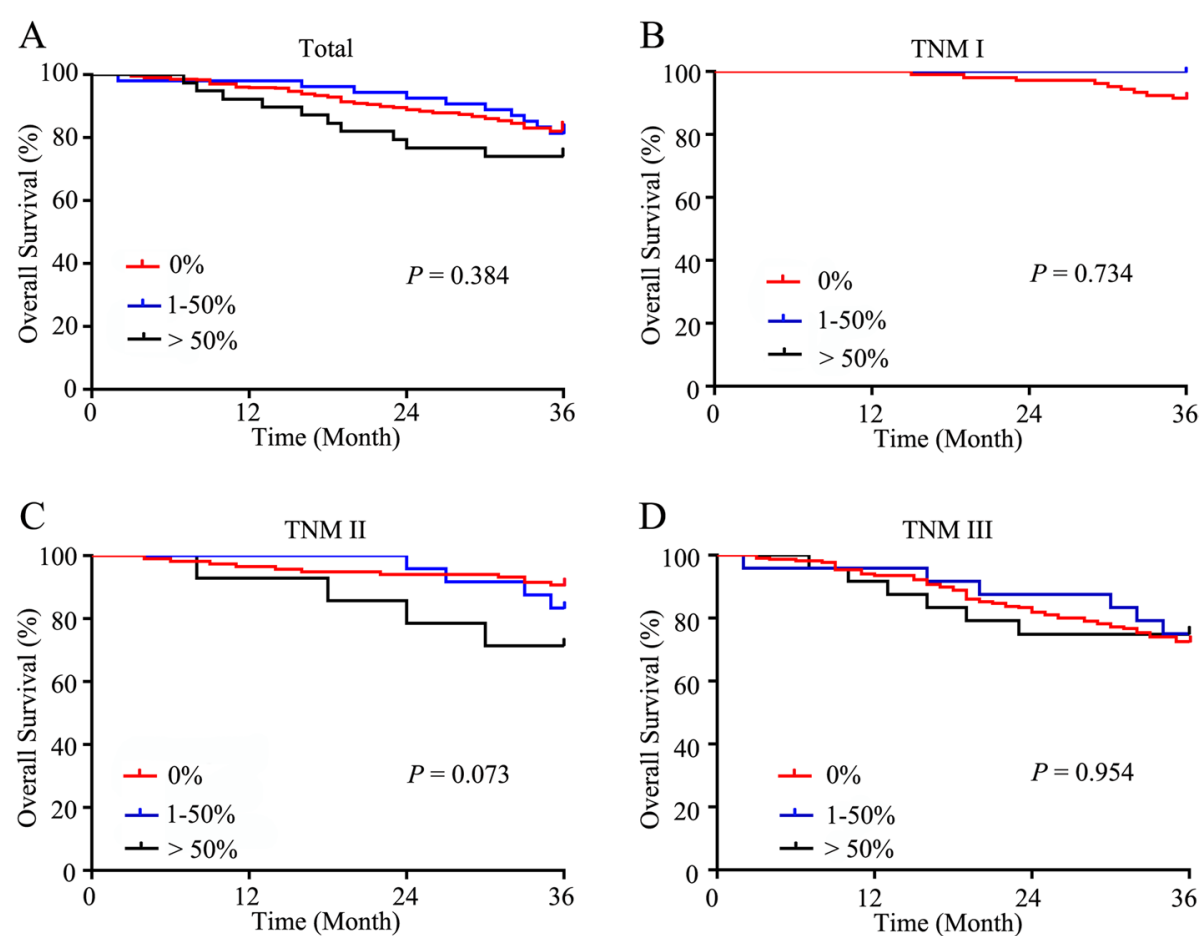

Fig. 1 Survival of patients in the AC group, the MAC group and the ACMC (1-50\%) group before matching. A. All involved patients. B. TNM stage I patients. C. TNM stage II patients. D. TNM stage III patients 
cells, regardless of extent, which typically show displacement and molding of the nucleus.

\section{Statistical analysis}

The data are presented as the mean and SD or as the median and range. For differences in categorical variables, chi-square analysis, Fisher exact test or KruskalWallis ANOVA test was performed where appropriate. Survival was depicted with Kaplan-Meier curves and compared using log-rank tests. Univariable and multivariable survival analyses using Cox regression models were performed to identify prognostic factors. Hazard ratios (HRs) were presented with 95\% confidence intervals (95\%CI). Propensity-score matched (PSM) analysis was conducted to minimize bias. The 1:1 matching process was performed by using the nearest neighbor matching method, with a maximum caliper width of 0.03 times the standard deviation of the logit (propensity score). Variables adjusted included gender, age, history of smoking and alcoholism, ASA class, comorbidities, preoperative
CEA, CA19-9, albumin, and HGB levels, occult blood status, operative factors and tumor factors. Receiveroperating characteristic (ROC) curve was used to identify the optimal cut-off value of $\mathrm{MC}$ ratio for prognostic prediction. At each ratio, the sensitivity and specificity for survival were determined and plotted, thereby generating a ROC curve. According to the $(0,1)$ criterion, the point on of the curve with the shortest distance to the coordinate $(0,1)$ was chosen as the cut-off value. Twosided $P \leq 0.050$ was considered statistically significant. All statistical analyses were performed using the SPSS software program (version 22.0 for Windows, IBM SPSS Statistics, IBM Corporation, Armonk, NY).

\section{Results}

Patient characteristics before matching

A total of 532 CRC patients were enrolled in this study. The clinicopathological features of these patients are shown in Table 1 . Mean age of the patients was $(64.51 \pm 12.09)$ years, including 315 males and 217

Table 2 Univariable and multivariable analysis for patients before matching

\begin{tabular}{|c|c|c|c|c|c|c|}
\hline \multirow[t]{2}{*}{ Parameters } & \multicolumn{3}{|c|}{ Univariable analysis } & \multicolumn{3}{|c|}{ Multivariable analysis } \\
\hline & HR & $95 \% \mathrm{Cl}$ & $P$ value & HR & $95 \% \mathrm{Cl}$ & $P$ value \\
\hline Gender Female vs. Male & 1.156 & $0.770-1.737$ & 0.485 & & & \\
\hline Age $<60$ years vs. $\geq 60$ years & 1.165 & $0.748-1.813$ & 0.499 & & & \\
\hline Smoking No vs. Yes & 1.197 & $0.769-1.863$ & 0.426 & & & \\
\hline Alcoholism No vs. Yes & 1.691 & $1.110-2.577$ & 0.015 & 1.482 & $0.963-2.281$ & 0.074 \\
\hline ASA class II vs. III & 0.982 & $0.601-1.605$ & 0.943 & & & \\
\hline Hypertension No vs. Yes & 0.777 & $0.491-1.230$ & 0.282 & & & \\
\hline Diabetes mellitus No vs. Yes & 1.145 & $0.661-1.984$ & 0.628 & & & \\
\hline CAD No vs. Yes & 0.668 & $0.310-1.440$ & 0.303 & & & \\
\hline Hepatitis No vs. Yes & 2.187 & $0.693-6.903$ & 0.182 & & & \\
\hline$C E A<5 \mathrm{ng} / \mathrm{ml}$ vs. $\geq 5 \mathrm{ng} / \mathrm{ml}$ & 2.372 & $1.599-3.519$ & $<0.001$ & 1.830 & $1.196-2.800$ & 0.005 \\
\hline CA19-9 <37U/ml vs. $\geq 37 \mathrm{U} / \mathrm{ml}$ & 2.259 & $1.406-3.629$ & 0.001 & 1.327 & $0.793-2.222$ & 0.281 \\
\hline Albumin $<40 \mathrm{~g} / \mathrm{dl}$ vs. $\geq 40 \mathrm{~g} / \mathrm{dl}$ & 0.947 & $0.633-1.417$ & 0.790 & & & \\
\hline $\mathrm{HGB}<110 \mathrm{~g} / \mathrm{L}$ vs. $\geq 110 \mathrm{~g} / \mathrm{L}$ & 1.475 & 0.874 .485 & 0.536 & & & \\
\hline Occult blood No vs. Yes & 1.013 & $0.671-1.530$ & 0.950 & & & \\
\hline Operation time $<3 \mathrm{~h}$ vs. $\geq 3 \mathrm{~h}$ & 0.894 & $0.603-1.325$ & 0.576 & & & \\
\hline Perioperative blood transfusion No vs. Yes & 1.047 & $0.665-1.647$ & 0.844 & & & \\
\hline Tumor location Right-sided vs. Left-sided & 0.900 & $0.475-1.706$ & 0.746 & & & \\
\hline Defunctioning stoma No vs. Yes & 0.915 & $0.128-6.561$ & 0.930 & & & \\
\hline Postoperative complication Absent vs. Present & 2.312 & $1.485-3.599$ & $<0.001$ & 1.589 & $0.978-2.582$ & 0.061 \\
\hline Differentiation Well/Moderate vs. Poor & 2.442 & $1.617-3.688$ & $<0.001$ & 1.698 & $1.083-2.663$ & 0.021 \\
\hline Signet-ring cell component Absent vs. Present & 6.603 & $2.082-20.938$ & 0.001 & 1.821 & $0.522-6.349$ & 0.347 \\
\hline Mucin No vs. Yes & 1.226 & $0.750-2.002$ & 0.416 & & & \\
\hline PNI Yes vs. No & 3.712 & $2.108-6.538$ & $<0.001$ & 2.389 & $1.314-4.344$ & 0.004 \\
\hline LVI Yes vs. No & 2.709 & $1.720-4.266$ & $<0.001$ & 1.600 & $0.977-2.619$ & 0.062 \\
\hline T stage $1 / 2$ vs. $3 / 4$ & 2.809 & $1.568-5.034$ & 0.001 & 1.417 & $0.757-2.655$ & 0.276 \\
\hline N stage 0 vs. $1 / 2$ & 2.905 & $1.875-4.500$ & $<0.001$ & 1.704 & $1.048-2.771$ & 0.032 \\
\hline TNM I/II vs. III & 3.829 & $2.230-6.576$ & $<0.001$ & 1.704 & $1.048-2.771$ & 0.032 \\
\hline
\end{tabular}

ASA American Society of Anesthesiologists, CAD coronary artery disease, CEA carcinoembryonic antigen, CA19-9 carbohydrate antigen 19-9, HGB hemoglobin, TNM tumor-lymph node-metastasis, LVI lymphovascular invasion, $P N I$ perineural invasion 
Table 3 Clinicopathological parameters for patients after matching

\begin{tabular}{|c|c|c|c|c|c|c|}
\hline Clinicopathological parameters & $\begin{array}{l}\text { Adenocarcinoma vs. } \\
\text { Mucinous component } \\
(1-100 \%)\end{array}$ & $P$ & $\begin{array}{l}\text { Adenocarcinoma vs. } \\
\text { Mucinous component } \\
(1-50 \%)\end{array}$ & $P$ & $\begin{array}{l}\text { Adenocarcinoma vs. } \\
\text { Mucinous adenocarcinoma } \\
(>50 \%)\end{array}$ & $P$ \\
\hline Gender & & 0.537 & & 0.418 & & 0.051 \\
\hline Female & $42 / 38$ & & $19 / 23$ & & $23 / 15$ & \\
\hline Male & $42 / 46$ & & $31 / 27$ & & $11 / 19$ & \\
\hline Age & & 0.606 & & 1.000 & & 0.318 \\
\hline$<60$ years & $22 / 25$ & & $10 / 10$ & & $11 / 15$ & \\
\hline$\geq 60$ years & $62 / 59$ & & $40 / 40$ & & $23 / 19$ & \\
\hline Smoking & & 0.694 & & 0.349 & & 0.709 \\
\hline No & $67 / 69$ & & $23 / 40$ & & $31 / 29$ & \\
\hline Yes & $17 / 15$ & & $14 / 10$ & & $3 / 5$ & \\
\hline Alcoholism & & 0.694 & & 0.410 & & 0.752 \\
\hline No & $67 / 70$ & & $38 / 43$ & & $29 / 27$ & \\
\hline Yes & $17 / 14$ & & $12 / 7$ & & $5 / 7$ & \\
\hline ASA class & & 0.860 & & 1.000 & & 1.000 \\
\hline$\|$ & $62 / 63$ & & $36 / 36$ & & $26 / 27$ & \\
\hline III & $22 / 21$ & & $14 / 14$ & & $8 / 7$ & \\
\hline Hypertension & & 0.733 & & 0.829 & & 0.582 \\
\hline No & $61 / 59$ & & $34 / 35$ & & $26 / 24$ & \\
\hline Yes & $23 / 25$ & & $16 / 15$ & & $8 / 10$ & \\
\hline Diabetes mellitus & & 0.679 & & 0.444 & & 1.000 \\
\hline No & $69 / 71$ & & $39 / 42$ & & $32 / 31$ & \\
\hline Yes & $15 / 13$ & & $11 / 8$ & & $2 / 3$ & \\
\hline CAD & & 0.618 & & 0.829 & & 1.000 \\
\hline No & $76 / 74$ & & $44 / 43$ & & $32 / 31$ & \\
\hline Yes & $8 / 10$ & & $6 / 7$ & & $2 / 3$ & \\
\hline Hepatitis & & 1.000 & & 1.000 & & 1.000 \\
\hline No & $82 / 82$ & & $49 / 49$ & & $33 / 33$ & \\
\hline Yes & $2 / 2$ & & $1 / 1$ & & $1 / 1$ & \\
\hline CEA & & 0.522 & & 0.091 & & 0.457 \\
\hline$<5 \mathrm{ng} / \mathrm{ml}$ & $55 / 51$ & & $37 / 29$ & & $19 / 22$ & \\
\hline$\geq 5 \mathrm{ng} / \mathrm{ml}$ & $29 / 33$ & & $16 / 21$ & & $15 / 12$ & \\
\hline CA19-9 & & 0.842 & & 0.790 & & 1.000 \\
\hline$<37 \mathrm{U} / \mathrm{ml}$ & $68 / 69$ & & $41 / 42$ & & $27 / 27$ & \\
\hline$\geq 37 \mathrm{U} / \mathrm{ml}$ & $16 / 15$ & & $9 / 8$ & & $7 / 7$ & \\
\hline Albumin & & 1.000 & & 0.548 & & 0.331 \\
\hline$<40 \mathrm{~g} / \mathrm{dl}$ & $41 / 41$ & & $24 / 27$ & & $18 / 14$ & \\
\hline$\geq 40 \mathrm{~g} / \mathrm{dl}$ & $43 / 43$ & & $26 / 23$ & & $16 / 20$ & \\
\hline HGB & & 0.504 & & 0.668 & & 0.110 \\
\hline$<110 \mathrm{~g} / \mathrm{L}$ & $24 / 28$ & & $17 / 15$ & & $7 / 13$ & \\
\hline$\geq 110 \mathrm{~g} / \mathrm{L}$ & $60 / 56$ & & $33 / 35$ & & $27 / 21$ & \\
\hline Occult blood & & 0.642 & & 0.841 & & 0.804 \\
\hline No & $36 / 39$ & & $24 / 25$ & & $13 / 14$ & \\
\hline Yes & $48 / 45$ & & $26 / 25$ & & $21 / 20$ & \\
\hline Operation time & & 0.642 & & 0.689 & & 0.215 \\
\hline$<3 h$ & $46 / 39$ & & $25 / 23$ & & $11 / 16$ & \\
\hline$\geq 3 h$ & $48 / 45$ & & $25 / 27$ & & 23/18 & \\
\hline Perioperative blood transfusion & & 0.452 & & 0.640 & & 0.109 \\
\hline No & $68 / 64$ & & $37 / 39$ & & $31 / 25$ & \\
\hline Yes & $16 / 20$ & & $13 / 17$ & & $3 / 9$ & \\
\hline
\end{tabular}


Table 3 (continued)

\begin{tabular}{|c|c|c|c|c|c|c|}
\hline Clinicopathological parameters & $\begin{array}{l}\text { Adenocarcinoma vs. } \\
\text { Mucinous component } \\
(1-100 \%)\end{array}$ & $P$ & $\begin{array}{l}\text { Adenocarcinoma vs. } \\
\text { Mucinous component } \\
(1-50 \%)\end{array}$ & $P$ & $\begin{array}{l}\text { Adenocarcinoma vs. } \\
\text { Mucinous adenocarcinoma } \\
(>50 \%)\end{array}$ & $P$ \\
\hline Tumor location & & 0.717 & & 1.000 & & 0.380 \\
\hline Right-sided & $19 / 21$ & & $12 / 12$ & & $6 / 9$ & \\
\hline Left-sided & $65 / 63$ & & $38 / 38$ & & $28 / 25$ & \\
\hline Defunctioning stoma & & 1.000 & & 1.000 & & 1.000 \\
\hline No & $83 / 83$ & & $49 / 49$ & & $34 / 34$ & \\
\hline Yes & $1 / 1$ & & $1 / 1$ & & $0 / 0$ & \\
\hline Postoperative complication & & 1.000 & & 0.790 & & 0.770 \\
\hline Absent & $68 / 68$ & & $41 / 42$ & & $27 / 26$ & \\
\hline Present & $16 / 16$ & & $9 / 8$ & & $7 / 8$ & \\
\hline Differentiation & & 0.872 & & 0.509 & & 0.625 \\
\hline Well+Moderate & $54 / 55$ & & $34 / 37$ & & 20/18 & \\
\hline Poor & $30 / 29$ & & $16 / 13$ & & $14 / 16$ & \\
\hline Signet-ring cell component & & 1.000 & & 1.000 & & 1.000 \\
\hline Absent & $83 / 83$ & & $49 / 50$ & & $34 / 33$ & \\
\hline Present & $1 / 1$ & & $1 / 0$ & & $0 / 1$ & \\
\hline PNI & & 1.000 & & 1.000 & & 1.000 \\
\hline No & $80 / 80$ & & $47 / 47$ & & $33 / 33$ & \\
\hline Yes & $4 / 4$ & & $3 / 3$ & & $1 / 1$ & \\
\hline LVI & & 0.223 & & 1.000 & & 0.105 \\
\hline No & $77 / 72$ & & $44 / 44$ & & $33 / 28$ & \\
\hline Yes & $7 / 12$ & & $6 / 6$ & & $1 / 6$ & \\
\hline Tstage & & 0.816 & & 0.779 & & 1.000 \\
\hline $1 / 2$ & $11 / 10$ & & $8 / 7$ & & $3 / 3$ & \\
\hline $3 / 4$ & $73 / 74$ & & $42 / 43$ & & $31 / 31$ & \\
\hline N stage & & 0.757 & & 0.230 & & 0.324 \\
\hline 0 & $38 / 40$ & & $22 / 28$ & & $16 / 12$ & \\
\hline $1 / 2$ & $46 / 44$ & & $28 / 22$ & & $18 / 22$ & \\
\hline TNM stage & & 0.757 & & 0.230 & & 0.324 \\
\hline$|-| \mid$ & $38 / 40$ & & $22 / 28$ & & $16 / 12$ & \\
\hline III & $46 / 44$ & & $28 / 22$ & & $18 / 22$ & \\
\hline
\end{tabular}

ASA American Society of Anesthesiologists, CAD coronary artery disease, CEA carcinoembryonic antigen, CA19-9 carbohydrate antigen 19-9, HGB hemoglobin, TNM tumor-lymph node-metastasis, LVI lymphovascular invasion, $P N I$ perineural invasion

females. Postoperative complication rate was $15.2 \%$ (81/532). As indicated in Table 1, MAC patients have a higher rate of $\mathrm{CA19}-9 \geq 37 \mathrm{U} / \mathrm{ml}(P=0.006)$, albu$\min <40 \mathrm{~g} / \mathrm{dl}(P=0.006), \mathrm{HGB}<110 \mathrm{~g} / \mathrm{L}(P=0.007)$, presence of occult blood $(P=0.011)$, right-sided location $(P<0.001)$, poor differentiation $(P<0.001)$, and advanced T stage $(P<0.001)$. Other parameters were similar among the patients in the AC group, the ACMC (1-50\%) group, and the MAC group $(P>0.05)$ (Table 1$)$.

The median duration of follow-up was 49 months (range, 2-170 months). The rate of patients treated with adjuvant chemotherapy was $54.32 \%$ (289/532 cases), including 236 in the AC group, 24 in the MAC group, and 29 in the ACMC (1-50\%) group. The 3-year OS rates of the patients with all TNM stages, TNM stage I, II, and III were similar among the AC group (82.00, 91.51, 90.68, and $72.56 \%)$, ACMC (1-50\%) group (81.48, 100, 83.33, and $75.00 \%)$, and MAC group $(74.11,100,71.43$, and 74.77\%) $(P>0.05)$ (Fig. 1).

\section{Univariable and multivariable analyses of possible prognostic factors before matching}

To identify potential risk factors for poor prognosis, univariable and multivariable analyses were conducted. The results showed that history of alcoholism $(\mathrm{HR}=1.691$, 95\% $\mathrm{CI}=1.110-2.577, \quad P=0.015), \quad \mathrm{CEA} \geq 5 \mathrm{ng} /$ $\mathrm{ml} \quad(\mathrm{HR}=2.372, \quad 95 \% \mathrm{CI}=1.599-3.519, \quad P<0.001)$, $\mathrm{CA} 19-9 \geq 37 \mathrm{U} / \mathrm{ml} \quad(\mathrm{HR}=2.259, \quad 95 \% \mathrm{CI}=1.406-3.629$, 
$P=0.001)$, postoperative complication $\quad(\mathrm{HR}=2.312$, 95\%CI $=1.485-3.599, \quad P<0.001)$, poor differentiation $(\mathrm{HR}=2.442, \quad 95 \% \mathrm{CI}=1.617-3.688, \quad P<0.001)$, signetring cell component $(\mathrm{HR}=6.603,95 \% \mathrm{CI}=2.082-20.938$, $P=0.001), \quad$ PNI $\quad(\mathrm{HR}=3.712, \quad 95 \% \mathrm{CI}=2.108-6.538$, $P<0.001), \quad$ LVI $\quad(\mathrm{HR}=2.709, \quad 95 \% \mathrm{CI}=1.720-4.266$, $P<0.001)$, advanced T stage $(\mathrm{HR}=2.809,95 \% \mathrm{CI}=1.568-$ $5.034, P=0.001), \mathrm{N}$ stage $(\mathrm{HR}=2.905,95 \% \mathrm{CI}=1.875-$ $4.500, P<0.001)$, and TNM stage $(\mathrm{HR}=3.829$, $95 \% \mathrm{CI}=2.230-6.576, \quad P<0.001)$ were risk factors for poor OS (Table 2). When further subjecting these factors into multivariable analysis, $\mathrm{CEA} \geq 5 \mathrm{ng} / \mathrm{ml}(\mathrm{HR}=1.830$, $95 \% \mathrm{CI}=1.196-2.800, \quad P=0.005)$, poor differentiation $(\mathrm{HR}=1.698,95 \% \mathrm{CI}=1.083-2.663, P=0.021)$, PNI
$(\mathrm{HR}=2.389,95 \% \mathrm{CI}=1.314-4.344, P=0.004)$, advanced $\mathrm{N}$ stage $(\mathrm{HR}=1.704,95 \% \mathrm{CI}=1.048-2.771, P=0.032)$, and TNM stage $(\mathrm{HR}=1.704,95 \% \mathrm{CI}=1.048-2.771$, $P=0.032)$ were identified as independent risk factors for poor OS (Table 2).

\section{PSM analysis of survival outcomes}

To account for potential imbalances, PSM analysis was conducted. As a result, 84 patients in the ACMC (1-50\%) group $(n=50)$ and the MAC group $(n=34)$ were matched with 84 patients in the AC group. Mean age of the 168 patients was $(65.30 \pm 12.74)$ years. The median duration of follow-up was 49 months (range, 4-168 months). The clinicopathological features of the matched patients were
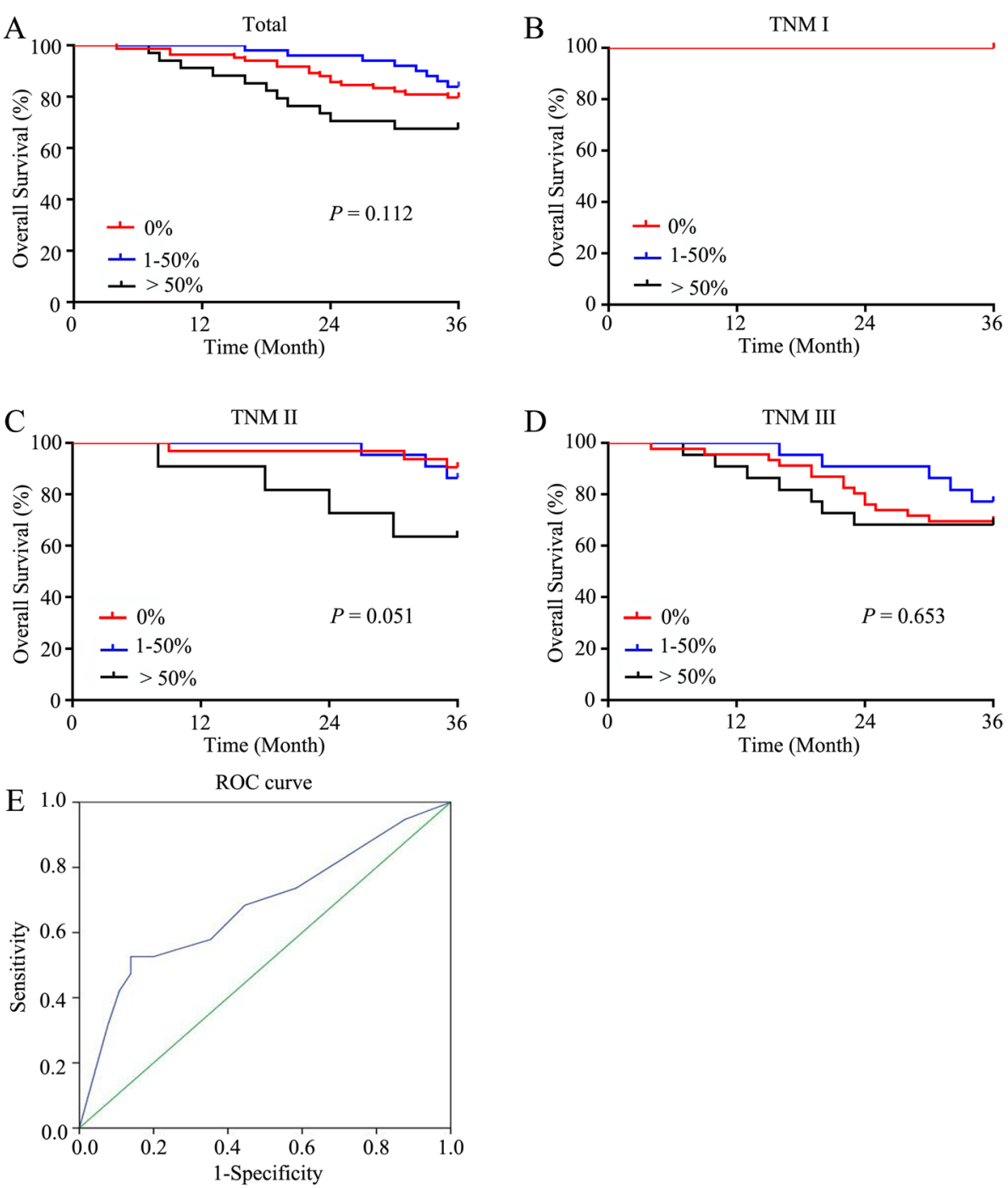

Fig. 2 Survival of patients in the AC group, the MAC group and the ACMC (1-50\%) group after matching. A. All involved patients. B. TNM stage I patients. C. TNM stage II patients. D. TNM stage III patients. E. ROC curve for determining the cut-off value of MC proportion for prognostic prediction 
similar (Table 3 ). The rate of patients receiving adjuvant chemotherapy was $58.33 \%$ (98/168 cases), including 49 in the AC group, 27 in the MAC group, and 22 in the ACMC (1-50\%) group. The 3-year OS rates of the patients with all TNM stages, TNM stage I, II, and III were similar in the AC group (79.76, 100, 90.63, and 69.57\%), ACMC (1-50\%) group $(84.00,100,86.36$, and $77.27 \%)$ and the MAC group $(67.65,100,63.64$, and $68.18 \%)(P>0.05)$ (Fig. 2A-D). To further to define the prognostic value of $\mathrm{MC}$ in CRC patients, ROC curve was adopted and $65 \%$ of mucinous area was determined as the optimal cut-off score (area under the curve $=0.677$ ) (Fig. 2E). To increase specificity, 70\% was used for the following analysis. As a result, patients with $A C M C>70 \%$ showed a much poorer survival compared with patients with ACMC (1-70\%) and $\mathrm{AC}$ patients $(47.37 \%$ vs. $86.15 \%$ vs. $79.76 \%, P<0.001)$ (Fig. 3A). In addition, the prognosis was also worse in patients with ACMC $>70 \%$ in TNM stage II patients (50.00\% vs. $88.00 \%$ vs. $90.63 \%, P=0.002)$ and TNM stage III patients $(45.46 \%$ vs. $81.82 \%$ vs. $69.57 \%, P=0.023)$ (Fig. 3C-D). However, the survival was similar in TNM stage I patients (100\% vs. $100 \%, P>0.999)$ (Fig. 3B).

\section{Univariable and multivariable analyses of possible prognostic factors after matching}

Possible prognostic factors were also analyzed by univariable and multivariable analyses after matching. As a result,
$\mathrm{MC}>70 \%(\mathrm{HR}=1.643,95 \% \mathrm{CI}=1.025-2.635, P=0.039)$, PNI $(\mathrm{HR}=2.969,95 \% \mathrm{CI}=1.049-8.400, P=0.040)$, LVI $(\mathrm{HR}=2.675,95 \% \mathrm{CI}=1.218-5.878, P=0.014)$, advanced $\mathrm{N}$ stage $(\mathrm{HR}=2.555,95 \% \mathrm{CI}=1.231-5.300, P=0.012)$, and advanced TNM stage $(\mathrm{HR}=2.555,95 \% \mathrm{CI}=1.231-$ $5.300, P=0.012$ ) were identified to be risk factors for poor OS (Table 4). Multivariable analysis found that advanced $\mathrm{N}$ stage $(\mathrm{HR}=2.210,95 \% \mathrm{CI}=1.035-4.719, P=0.041)$ and TNM stage $(\mathrm{HR}=2.210,95 \% \mathrm{CI}=1.035-4.719, P=0.041)$ were independent risk factors for poor OS (Table 4).

\section{Discussion}

MAC has different clinicopathological features compared with AC $[2,8]$. Consistently with previous reports $[2,8,9]$, our data revealed that MAC was associated with higher rate of right-sided location, poor differentiation, advanced $\mathrm{T}$ stage before matching, which indicated that MAC is more advanced at diagnosis. In addition, our results showed that MAC patients have a higher rate of albumin $<40 \mathrm{~g} / \mathrm{dl}, \mathrm{HGB}<110 \mathrm{~g} / \mathrm{L}$ and presence of occult blood, these parameters were seldom analyzed in previous studies. Our data suggested that MAC patients need more nutritional support and improvement in general conditions prior to surgery.

The survival of MAC patients or ACMC (1-50\%) patients has always been controversial in previous studies
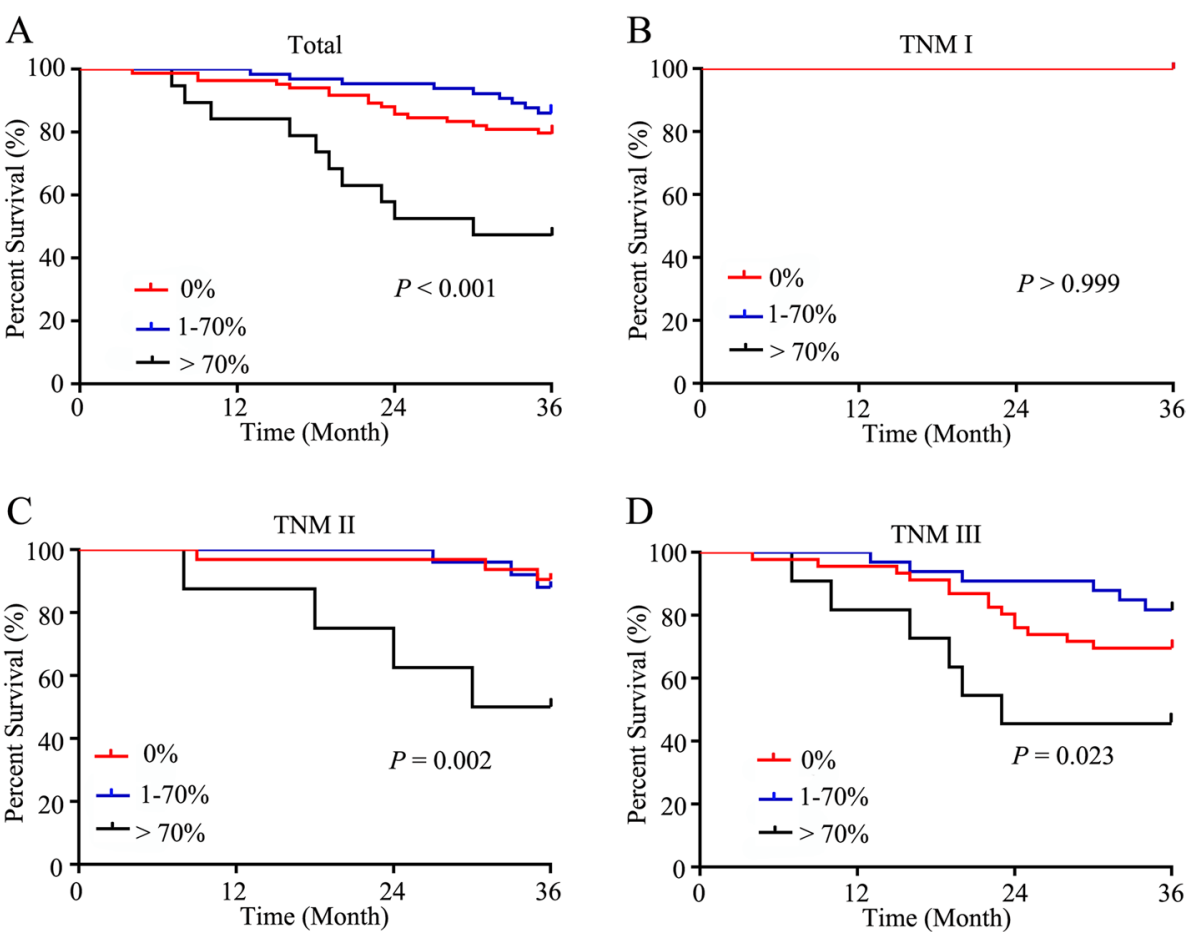

Fig. 3 Survival of patients in the AC group, the ACMC (> 70\%) group and the ACMC (1-70\%) group after matching. A. All involved patients. B. TNM stage I patients. C. TNM stage II patients. D. TNM stage III patients 
Table 4 Univariable and multivariable analysis for patients after matching

\begin{tabular}{|c|c|c|c|c|c|c|}
\hline \multirow[t]{2}{*}{ Parameters } & \multicolumn{3}{|c|}{ Univariable analysis } & \multicolumn{3}{|c|}{ Multivariable analysis } \\
\hline & HR & $95 \% \mathrm{Cl}$ & $P$ value & HR & $95 \% \mathrm{Cl}$ & $P$ value \\
\hline Gender Female vs. Male & 1.301 & $0.671-2.524$ & 0.436 & & & \\
\hline Age $<60$ years vs. $\geq 60$ years & 0.626 & $0.317-1.235$ & 0.177 & & & \\
\hline Smoking No vs. Yes & 0.656 & $0.255-1.678$ & 0.381 & & & \\
\hline Alcoholism No vs. Yes & 1.076 & $0.471-2.456$ & 0.862 & & & \\
\hline ASA class II vs. III & 0.790 & $0.360-1.733$ & 0.556 & & & \\
\hline Hypertension No vs. Yes & 0.689 & $0.314-1.512$ & 0.353 & & & \\
\hline Diabetes mellitus No vs. Yes & 0.994 & $0.414-2.389$ & 0.990 & & & \\
\hline CAD No vs. Yes & 1.045 & $0.369-2.954$ & 0.935 & & & \\
\hline Hepatitis No vs. Yes & 1.199 & $0.164-8.752$ & 0.858 & & & \\
\hline CEA $<5 \mathrm{ng} / \mathrm{ml}$ vs. $\geq 5 \mathrm{ng} / \mathrm{ml}$ & 1.841 & $0.958-3.539$ & 0.067 & & & \\
\hline CA19-9 <37 U/ml vs. $\geq 37 \mathrm{U} / \mathrm{ml}$ & 1.302 & $0.593-2.857$ & 0.510 & & & \\
\hline Albumin $<40 \mathrm{~g} / \mathrm{dl}$ vs. $\geq 40 \mathrm{~g} / \mathrm{dl}$ & 1.241 & $0.643-2.394$ & 0.520 & & & \\
\hline $\mathrm{HGB}<110 \mathrm{~g} / \mathrm{L}$ vs. $\geq 110 \mathrm{~g} / \mathrm{L}$ & 1.611 & $0.734-3.536$ & 0.234 & & & \\
\hline Occult blood No vs. Yes & 1.537 & $0.778-3.034$ & 0.216 & & & \\
\hline Operation time $<3 \mathrm{~h}$ vs. $\geq 3 \mathrm{~h}$ & 1.483 & $0.751-2.927$ & 0.256 & & & \\
\hline Perioperative blood transfusion No vs. Yes & 0.855 & $0.375-1.953$ & 0.711 & & & \\
\hline Tumor location Right-sided vs. Left-sided & 1.364 & $0.597-3.113$ & 0.461 & & & \\
\hline Defunctioning stoma No vs. Yes & 0.049 & $0.000-16,660.644$ & 0.642 & & & \\
\hline Postoperative complication Absent vs. Present & 1.504 & $0.707-3.189$ & 0.289 & & & \\
\hline Differentiation Well/Moderate vs. Poor & 1.267 & $0.648-2.476$ & 0.490 & & & \\
\hline Signet-ring cell component Absent vs. Present & 2.842 & $0.388-20.785$ & 0.304 & & & \\
\hline Mucin No vs. Yes & 1.126 & $0.585-2.167$ & 0.722 & & & \\
\hline Mucin component $0 \%$ vs. $1-70 \%$ vs. $>70 \%$ & 1.643 & $1.025-2.635$ & 0.039 & 1.550 & $0.958-2.507$ & 0.074 \\
\hline PNI Yes vs. No & 2.969 & $1.049-8.400$ & 0.040 & 2.105 & $0.713-6.218$ & 0.178 \\
\hline LVI Yes vs. No & 2.675 & $1.218-5.878$ & 0.014 & 1.687 & $0.721-3.944$ & 0.228 \\
\hline T stage $1 / 2$ vs. $3 / 4$ & 5.531 & $0.758-40.375$ & 0.092 & & & \\
\hline N stage 0 vs. 1/2 & 2.555 & $1.231-5.300$ & 0.012 & 2.210 & $1.035-4.719$ & 0.041 \\
\hline TNM I/II vs. III & 2.555 & $1.231-5.300$ & 0.012 & 2.210 & $1.035-4.719$ & 0.041 \\
\hline
\end{tabular}

ASA American Society of Anesthesiologists, CAD coronary artery disease, CEA carcinoembryonic antigen, CA19-9 carbohydrate antigen 19-9, HGB hemoglobin, TNM tumor-lymph node-metastasis, LVI lymphovascular invasion, $P N I$ perineural invasion

$[2,4,8-24]$. The retrospective nature of these studies may be an essential factor leading to the difference. Two PSM studies, minimizing confounding factors statistically, discovered that MAC was a prognostic factor in TNM stage II patients $[6,25]$. This study found that the survival of patients in the AC group, the ACMC (1-50\%) group, and the MAC group were similar both before matching and after matching. However, MAC exhibited a relatively low 3 -year OS compared to ACMC (1-50\%) and AC in TNM stage II patients after matching, though no statistical significance was detected $(P=0.051)$. However, the detailed mechanisms of MAC patients with TNM stage II exhibited poorer survival need further investigation. It has been recommended that adjuvant chemotherapy should be routinely performed for patients with stage II MAC, and special attention should be paid during follow-up because of the risk of peritoneal or local recurrence [25].
To further define the clinical significance of $\mathrm{MC}$ in CRC. Our study re-evaluated the MC proportion more accurately and $70 \%$ was found to be a cut-off value for predicting prognosis, which is rarely reported in previous studies. Patients with $\mathrm{MC}>70 \%$ displayed a much poorer 3-year OS compared with patients with ACMC 1-70\% and $\mathrm{AC}$ patients both in all patients and stage-matched (TNM stage II and stage III) patients. In addition, $\mathrm{MC}>70 \%$ was demonstrated to be a risk factor of poor OS in univariable analysis, though not an independent risk factor to multivariable analysis. Thus, the effect of mucin on survival may be associated with its proportion in the lesion, and $\mathrm{MC}>70 \%$ may serve as a biomarker for poor prognosis.

To better understand the cause of diverse clinical behaviors, numerous studies have focused on discovering the gene expression profiling in MAC [26-29]. 
Li et al. have detected that the combined mutation frequency of the two key factors of the EGFR signaling pathway, KRAS and BRAF, in the CRCs with and without MC was 95.9 and $52.1 \%$, respectively. The desregulated EGFR pathway plays a pivotal role in the development of ACMC, irrespective of the percentage [26]. Besides, low frequency of mutations in the p53 gene or overexpression of $\mathrm{p} 53$ protein and loss of heterozygosity in the DCC gene have been reported [30, 31]. Genome-wide analysis found that MAC displayed 182 upregulated and 135 downregulated genes compared with AC [29]. The most upregulated genes included those involved in cellular differentiation and mucin metabolism, and altered biologic pathways included those associated with mucin substrate metabolism, amino acid metabolism, and the mitogen-activated protein kinase cascade [29]. Consistently, MUC2, which is one of the glycosylated proteins, was reported to be overexpressed in MAC [32, 33]. In addition, MAC overexpresses both TYMS and GSTP1, biomarkers indicating resistance to 5-FU and oxaliplatin [34]. These findings may partially illustrate the different phenotypes of MAC.

In conclusion, this study detected that MAC is usually diagnosed as an advanced stage. MAC patients have a similar survival with AC patients and ACMC (1-50\%) patients before and after matching. Mucin accounting for more than $70 \%$ in the lesion is a more valuable cut-off score of predicting poor survival. Patients with $\mathrm{MC}>70 \%$ should be given more clinical attention. However, data was retrospectively reviewed in this study, although PSM was conducted to adjust for known confounding factors, some degree of selection bias cannot be ruled out.

\section{Abbreviations}

MC: Mucinous component; AC: Colorectal adenocarcinoma; PSM: Propensity score matching; ROC: Receiver-operating characteristic; OS: Overall survival; MAC: Mucinous adenocarcinoma; HR: Hazard Ratio; CRC: Colorectal cancer; WHO: World Health Organization; ASA: The American Society of Anesthesiologists; CAD: Coronary artery disease; CEA: Carcinoembryonic antigen; HGB: Hemoglobin; PNI: Perineural invasion; LVI: Lymphovascular invasion; ACMC: With colorectal $\mathrm{AC}$ and $\mathrm{AC}$ with MC.

\section{Acknowledgements}

Not applicable.

\section{Authors' contributions}

LJX, QS and FY designed the research study. CWY, HY and LLC analyzed the data and wrote the paper. CWY, HY, LLC, RL, WS and WGY collected clinical date and followed up. LLC and QS reviewed the pathological slides and calculated the proportion of mucus in the tumor. All authors have read and approved the manuscript.

\section{Funding}

No funding was obtained for this study.

\section{Availability of data and materials}

The datasets used or analysed during the current study are available from the corresponding author on reasonable request.

\section{Declarations}

Ethics approval and consent to participate

This study was approved by the First Affiliated Hospital of Shandong First Medical University \& Shandong Provincial Qianfoshan Hospital Institutional Review Board. All the experiment protocol for involving human data was in accordance with the guidelines of national in the manuscript. The study obtained the written consent of all participants.

\section{Consent for publication}

Not applicable.

\section{Competing interests}

Nothing to declare.

\section{Author details}

${ }^{1}$ Department of General Surgery, Shandong Provincial Qianfoshan Hospital, Weifang Medical University, Key Laboratory of Metabolism and Gastrointestinal Tumor, the First Affiliated Hospital of Shandong First Medical University, Key Laboratory of Laparoscopic Technology, the First Affiliated Hospital of Shandong First Medical University, Shandong Medicine and Health Key Laboratory of General Surgery, Weifang 261000, Shandong, China. ${ }^{2}$ Department of General Surgery, The First Affiliated Hospital of Shandong First Medical University \& Shandong Provincial Qianfoshan Hospital, Key Laboratory of Metabolism and Gastrointestinal Tumor, the First Affiliated Hospital of Shandong First Medical University, Key Laboratory of Laparoscopic Technology, the First Affiliated Hospital of Shandong First Medical University, Shandong Medicine and Health Key Laboratory of General Surgery, Jinan 250000, Shandong, China. ${ }^{3}$ Department of Pathology, Jinan Central Hospital Affiliated to Shandong First Medical University, Jinan 250000, Shandong, China. ${ }^{4}$ Department of Pathology, The First Affiliated Hospital of Shandong First Medical University \& Shandong Provincial Qianfoshan Hospital, Shandong Medicine and Health Key Laboratory of Clinical Pathology, Shandong Lung Cancer Institute, Shandong Institute of Nephrology, Jinan, China.

Received: 16 June 2021 Accepted: 15 November 2021

Published online: 01 December 2021

\section{References}

1. Dekker E, Tanis PJ, Vleugels JLA, Kasi PM, Wallace MB. Colorectal cancer. Lancet. 2019;394(10207):1467-80.

2. Verhulst J, Ferdinande L, Demetter P, Ceelen W. Mucinous subtype as prognostic factor in colorectal cancer: a systematic review and metaanalysis. J Clin Pathol. 2012;65(5):381-8.

3. Nagtegaal ID, Odze RD, Klimstra D, Paradis V, Rugge M, Schirmacher P, et al. The 2019 WHO classification of tumours of the digestive system. Histopathology. 2020;76(2):182-8.

4. Chew MH, Yeo SA, Ng ZP, Lim KH, Koh PK, Ng KH, et al. Critical analysis of mucin and signet ring cell as prognostic factors in an Asian population of 2,764 sporadic colorectal cancers. Int J Color Dis. 2010;25(10):1221-9.

5. Kelemen LE, Köbel M. Mucinous carcinomas of the ovary and colorectum: different organ, same dilemma. Lancet Oncol. 2011;12(11):1071-80.

6. Kanda M, Oba K, Aoyama T, Kashiwabara K, Mayanagi S, Maeda H, et al. Clinical signatures of mucinous and poorly differentiated subtypes of colorectal adenocarcinomas by a propensity score analysis of an independent patient database from three phase III trials. Dis Colon Rectum. 2018;61(4):461-71.

7. Ott C, Gerken M, Hirsch D, Fest P, Fichtner-Feigl S, Munker S, et al. Advanced mucinous colorectal Cancer: epidemiology, prognosis and efficacy of chemotherapeutic treatment. Digestion. 2018;98(3):143-52.

8. Nitsche U, Zimmermann A, Späth C, Müller T, Maak M, Schuster T, et al. Mucinous and signet-ring cell colorectal cancers differ from classical adenocarcinomas in tumor biology and prognosis. Ann Surg. 2013;258(5):775-82 discussion 782-773.

9. Hosseini S, Bananzadeh AM, Salek R, Zare-Bandamiri M, Kermani AT, Mohammadianpanah M. Prognostic significance of mucinous histologic subtype on oncologic outcomes in patients with colorectal Cancer. Ann Coloproctol. 2017;33(2):57-63. 
10. Tarantino I, Hüttner FJ, Warschkow R, Schmied BM, Diener MK, Ulrich A. Prognostic relevance of mucinous subtype in a population-based propensity score analysis of 40,083 rectal Cancer patients. Ann Surg Oncol. 2016;23(5):1576-86.

11. Negri FV, Wotherspoon A, Cunningham D, Norman AR, Chong G, Ross PJ. Mucinous histology predicts for reduced fluorouracil responsiveness and survival in advanced colorectal cancer. Ann Oncol. 2005;16(8):1305-10.

12. Li Z-P, Liu X-Y, Kao X-M, Chen Y-T, Han S-Q, Huang M-X, et al. Clinicopathological characteristics and prognosis of colorectal mucinous adenocarcinoma and nonmucinous adenocarcinoma: a surveillance, epidemiology, and end results (SEER) population-based study. Ann Transl Med. 2020;8(5):205

13. Kanemitsu Y, Kato T, Hirai T, Yasui K, Morimoto T, Shimizu Y, et al. Survival after curative resection for mucinous adenocarcinoma of the colorectum. Dis Colon Rectum. 2003;46(2):160-7.

14. Mekenkamp LJ, Heesterbeek KJ, Koopman M, Tol J, Teerenstra S, Venderbosch $\mathrm{S}$, et al. Mucinous adenocarcinomas: poor prognosis in metastatic colorectal cancer. Eur J Cancer. 2012;48(4):501-9.

15. Nitsche U, Friess H, Agha A, Angele M, Eckel R, Heitland W, et al. Prognosis of mucinous and signet-ring cell colorectal cancer in a population-based cohort. J Cancer Res Clin Oncol. 2016;142(11):2357-66.

16. Ooki A, Akagi K, Yatsuoka T, Asayama M, Hara H, Yamamoto G, et al. Inverse effect of mucinous component on survival in stage III colorectal cancer. J Surg Oncol. 2014;110(7):851-7.

17. Catalano V, Loupakis F, Graziano F, Bisonni R, Torresi U, Vincenzi B, et al. Prognosis of mucinous histology for patients with radically resected stage II and III colon cancer. Ann Oncol. 2012;23(1):135-41.

18. Inamura K, Yamauchi M, Nishihara R, Kim SA, Mima K, Sukawa Y, et al. Prognostic significance and molecular features of signet-ring cell and mucinous components in colorectal carcinoma. Ann Surg Oncol. 2014;22(4):1226-35.

19. Song BR, Xiao CC, Wu ZK. Predictors of lymph node metastasis and prognosis in pT1 colorectal Cancer patients with signet-ring cell and mucinous adenocarcinomas. Cell Physiol Biochem. 2017;41(5):1753-65.

20. Kang H, O'Connell JB, Maggard MA, Sack J, Ko CY. A 10-year outcomes evaluation of mucinous and signet-ring cell carcinoma of the colon and rectum. Dis Colon Rectum. 2005;48(6):1161-8.

21. Gonzalez RS, Cates JMM, Washington K. Associations among histological characteristics and patient outcomes in colorectal carcinoma with a mucinous component. Histopathology. 2019;74(3):406-14.

22. Langner C, Harbaum L, Pollheimer MJ, Kornprat P, Lindtner RA, Schlemmer $A$, et al. Mucinous differentiation in colorectal cancer - indicator of poor prognosis? Histopathology. 2012;60(7):1060-72.

23. Thota R, Fang $X$, Subbiah S. Clinicopathological features and survival outcomes of primary signet ring cell and mucinous adenocarcinoma of colon: retrospective analysis of VACCR database. J Gastrointest Oncol. 2014;5(1):18-24

24. Maeda Y, Sadahiro S, Suzuki T, Haruki Y, Nakamura N. Significance of the mucinous component in the histopathological classification of colon cancer. Surg Today. 2015;46(3):303-8.

25. Wang L, Hirano Y, Heng G, Ishii T, Kondo H, Hara K, et al. Mucinous adenocarcinoma as a high-risk factor in stage II colorectal Cancer: a propensity score-matched study from Japan. Anticancer Res. 2020;40(3):1651-9.

26. Li X, Sun K, Liao X, Gao H, Zhu H, Xu R. Colorectal carcinomas with mucinous differentiation are associated with high frequent mutation of KRAS or BRAF mutations, irrespective of quantity of mucinous component. BMC Cancer. 2020;20(1):400.

27. Kim H, Kim B-H, Lee D, Shin E. Genomic alterations in signet ring and mucinous patterned colorectal carcinoma. Pathol Res Pract. 2019;215(10):152566.

28. Andrici J, Farzin M, Sioson L, Clarkson A, Watson N, Toon CW, et al. Mismatch repair deficiency as a prognostic factor in mucinous colorectal cancer. Mod Pathol. 2016;29(3):266-74.

29. Melis M, Hernandez J, Siegel EM, McLoughlin JM, Ly QP, Nair RM, et al. Gene expression profiling of colorectal mucinous adenocarcinomas. Dis Colon Rectum. 2010;53(6):936-43.

30. Campo E, de la Calle-Martin O, Miquel R, Palacin A, Romero M, Fabregat $\checkmark$, et al. Loss of heterozygosity of p53 gene and p53 protein expression in human colorectal carcinomas. Cancer Res. 1991;51(16):4436-42.

31. Hedrick L, Cho KR, Fearon ER, Wu TC, Kinzler KW, Vogelstein B. The DCC gene product in cellular differentiation and colorectal tumorigenesis. Genes Dev. 1994;8(10):1174-83.

32. Hanski C, Hofmeier M, Schmitt-Gräff A, Riede E, Hanski ML, Borchard F, et al. Overexpression or ectopic expression of MUC2 is the common property of mucinous carcinomas of the colon, pancreas, breast, and ovary. J Pathol. 1997;182(4):385-91.

33. Ho SB, Niehans GA, Lyftogt C, Yan PS, Cherwitz DL, Gum ET, et al. Heterogeneity of mucin gene expression in normal and neoplastic tissues. Cancer Res. 1993;53(3):641-51.

34. Glasgow SC, Yu J, Carvalho LP, Shannon WD, Fleshman JW, McLeod HL. Unfavourable expression of pharmacologic markers in mucinous colorectal cancer. Br J Cancer. 2005;92(2):259-64.

\section{Publisher's Note}

Springer Nature remains neutral with regard to jurisdictional claims in published maps and institutional affiliations.
Ready to submit your research? Choose BMC and benefit from:

- fast, convenient online submission

- thorough peer review by experienced researchers in your field

- rapid publication on acceptance

- support for research data, including large and complex data types

- gold Open Access which fosters wider collaboration and increased citations

- maximum visibility for your research: over $100 \mathrm{M}$ website views per year

At BMC, research is always in progress.

Learn more biomedcentral.com/submissions 